\title{
Fracture aperture reconstruction and determination of hydrological properties: a case study at Draix (French Alps)
}
A. Neuville ${ }^{1,2}$
R. Toussaint ${ }^{1,2}$
J. Schmittbuhl ${ }^{1,2}$

February 25, 2010

\begin{abstract}
amelie.neuville@eost.u-strasbg.fr
renaud.toussaint@eost.u-strasbg.fr

jean.schmittbuhl@eost.u-strasbg.fr
\end{abstract}

Submitted to HP - special issue paper, with the reference S147 Clay Shales and Clayey Sediments

\begin{abstract}
We propose two techniques for fracture aperture reconstruction. The first one is a correlation technique that estimates the normal aperture or the tangential shift across a discontinuity whose sides present geometrical similarities. The only required material is a pair of appropriately controlled images of each side. Here the images are maps of the corresponding side's topography, obtained from laser profilometry. Assuming a purely normal opening, it is possible, from two corresponding sides of a given discontinuity in a core log, to infer the precise geometry of the in situ aperture. The second technique allows to retrieve the three dimensional geometry of a sealed discontinuity from non independent topography measurements of both sides. Both techniques are applied to discontinuities extracted from a core drilled down to $20 \mathrm{~m}$ in a fractured marl formation at Draix (French Alps). The probability density functions of the aperture of the sealed and open discontinuities are Gaussian. At the sample scale, the sealed fracture aperture is self-affine while the open one shows a crossover from an self-affine
\end{abstract}

\footnotetext{
${ }^{1}$ EOST, Université de Strasbourg, France

${ }^{2}$ Institut de Physique du Globe de Strasbourg, UMR CNRS UdS 7516, 5 rue René Descartes, 67087 Strasbourg Cedex, France
} 
regime at very small scales to an uncorrelated regime at largest scales. Those scaling laws are extrapolated at the scale of the whole formation, and the hydraulic properties of the Draix fractured bedrock can be estimated. In some cases, the aperture roughness has a negligible influence on its hydraulic behavior, and the large scale permeability can directly be estimated. In others, flow simulations are necessary to render for the roughness effect. We perform these simulations using a finite difference method under the lubrication approximation at low Reynolds number. The overall estimated permeability is significant $\left(10^{-9}-10^{-8} \mathrm{~m}^{2}\right)$, consistently with some previous indirect inferences.[PB: RESUME TROP LONG - LIMITE A 250 MOTS ACTUEL: 276MOTS]

\section{Introduction}

Many physical processes occurring in fractured rocks depend on the individual fracture apertures: for instance the hydraulic flow (e.g. Brown (1987); Tsang and Tsang (1987); Pyrak-Nolte et al. (1988); Zimmerman and Bodvarsson (1996); Méheust and Schmittbuhl (2001); Drazer and Koplik (2002); Auradou et al. (2005, 2006); Schmittbuhl et al. (2008)), the tracer dispersion (e.g. Drazer and Koplik (2002); Roux et al. (1998); Plouraboué et al. (1998)) or the heat transfer (e.g. Walkup and Horne (1985); Muralidhar (1990); Neuville et al. (2009a)). It is therefore important to be able to reconstitute the volumetric geometry of apertures, knowing both the average opening and the fracture roughness. The hydraulic properties are notably highly influenced by the ratio between roughness amplitude and mean aperture (e.g. Brown (1987); Méheust and Schmittbuhl (2001); Al-Yaarubi et al. $(2005))$.

This paper shows how to reconstruct, at laboratory scale, the aperture of some types of open fractures and the thickness of veins, in order to estimate the hydraulic behavior in a fractured bedrock. The studied discontinuities are extracted from a core drilled in the fractured marl of Draix (French Alps), an experimental site exploited to understand landslide processes (Fressard et al., 2009). At laboratory scale, aperture reconstruction of fracture has previously been explored in various ways depending if the fracture fully intercepts the sample (providing two sample pieces) or not. Some of the methods allow to obtain the aperture fluctuations directly: for instance X-ray computer tomography measurements (e.g. Noiriel (2005)) or resin injection into the fracture (e.g. Gentier and Hopkins (1997); Hakami and Larsson (1996)). In Hakami and Larsson (1996) the sample was then sliced perpendicularly to the fracture and the aperture was measured on each slide using images obtained from an optical microscope. If both sides of the fracture are separated, 
the aperture fluctuations can also be obtained indirectly, by measuring the topography of facing fracture surfaces whose relative position is ensured by a common reference frame (e.g. the boundaries of the sample). For instance, it was done by using transparent casts of each surfaces, whose morphology can be obtained through light attenuation properties (e.g. Isakov et al. (2001)), and also by measuring the morphology of surfaces using mechanical or laser profilers (e.g.Vicente Silvestre et al. (2002); Sharifzadeh et al. (2008); Lee and Cho (2002); Sakaguchi et al. (2008)). In some of the previously cited studies, the average aperture is either a priori known, especially when the sample fully embodies the fracture, or arbitrarily fixed: for instance when both fracture sides are assumed to be in contact. When the sample is entirely intercepted by the discontinuity, providing two separated sides whose relative position is unknown, the average aperture is tricky to estimate. Such open discontinuities, showing no contact at sample scale between both sides can typically be found in samples extracted from a core drilled in fractured bedrocks (e.g. Lofi et al. (2009); Genter and Traineau (1996)). For a sealed discontinuity, the situation is different. Boundary geometry can be directly measured if the embedding material can be removed. However note, even in this case, that the volumetric geometry is lost when both surface elevations are not measured in a common reference frame. In section 2 of this paper, we detail two methods, respectively dedicated to open and sealed discontinuities, allowing the reconstruction of both the average aperture and the roughness variation of a discontinuity. The first method requires the analyze of the correlations between the surfaces, provided that these correlations exist. For a sealed discontinuity, the relative positioning is ensured by attaching the sample to a specific frame when measuring each surface topography. Both methods are illustrated on experimental discontinuities whose surface elevations were previously measured with a laser profiler (Neuville et al. (2009b), hereafter referred as paper 1), providing an accurate knowledge of each surface topography. In section 3 a statistical study of the fluctuations of the resulting apertures is presented, and the scaling laws governing the aperture roughness are determined. In section 4, the hydraulic behavior of the various discontinuities is determined and the observed scaling laws are used to obtain the large scale hydraulic properties of the formation. 


\section{Aperture reconstruction}

\subsection{Reconstruction of the aperture of an open disconti- nuity with correlated sides}

\subsubsection{Topography of the sides at zero relative displacement}

Let us consider a brittle elastic fracturing that creates two sides with complementary morphologies (or asperities). If the displacement induced by the fracturing is zero, then each surface asperity is in contact with the corresponding asperity on the other surface, as shown in Fig. 1a. Note that this state might be fictitious if the relative displacement occurs at the same time as the fracturing. In practice, during the bedrock history, the discontinuity morphology might be modified due to chemo-mechanical processes, hence correlation between both surfaces may disappear. Let us consider that despite a probable alteration of the fracture, enough complementary asperities may still be observed.

\subsubsection{Present displacement indicated by the borehole boundaries}

When the drilling is performed, the intercept between the drill and both sides of the fracture is very likely to provide information about the relative position of the fracture sides at the drilling time. Both complementary sides might have been shifted relatively to each other. Handling the sample, and moving both sides relatively to each other, one can - in principle - restore contact between the complementary asperities (i.e., as defined above, the state of the fracture at zero displacement). In general, once the asperities are in contact, the boundaries of the facing sides no longer fit. When the fracture is not perpendicular to the cylindrical core axis, the intersection between the discontinuity mean plane and the boundary of the drill provides surfaces with elliptic boundaries. This only assumes that $\beta \neq 0$, where $\beta$ is defined as the angle between the normal to the mean discontinuity plane and the core axis (see Fig. 1). Let $\vec{\delta}$ be the projection of the true vector displacement field between surfaces, in the fracture mean plane (defined here as the plane fitted by a least mean square method of the surface topography). It can be measured from the shift of the elliptical boundaries once the complementary asperities are in contact. The vector field $\vec{\delta}$, that will further be called the in-plane shift, allows to reconstitute the true field of relative displacements of the fracture sides, under some simple geometrical assumptions. 


\subsubsection{Displacement history}

If the fracture opened in shear mode, i.e. with relative displacement parallel to the average plane, then $\vec{\delta}$ is uniform and its norm directly provides the shear value. Let us now consider the other end-member, i.e. a fracture that opened in mode I, whose sides moved along a direction normal to the mean plane. When the axis of the drill is not perpendicular to the mean discontinuity plane $(\beta \neq 0)$, the more the fracture is open, the more the facing patterns will appear shifted once the core is extracted from the drill (Fig. 1b). In this case, the field $\vec{\delta}$ is also uniform. Knowing the angle $\beta$ and the norm $\delta$ (Fig. 2), the average aperture $A$, measured in the direction perpendicular to the mean discontinuity plane, can be deduced: $A=\delta / \tan \beta$. The angle $\beta$ can be measured directly on the considered discontinuity using an inclinometer, once the core is extracted from the soil. Moreover this measurement allows to check if both sides of the open discontinuity are indeed parallel on average, as it should indeed be for pure mode I opening. In the next part, we show how $\vec{\delta}$ may be evaluated.

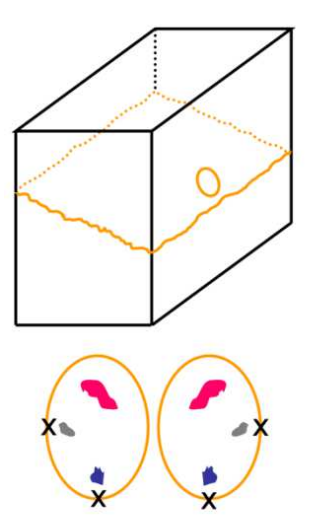

(a)

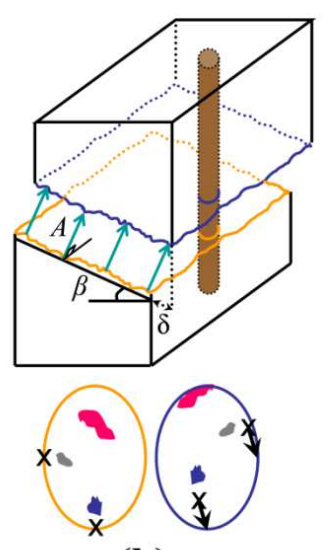

(b)

Figure 1: Comparison of the elliptic intercept of a drill before and after a normal shift of a discontinuity. In (b), the location of the elliptic boundary of the core with respect to the similar facing patterns (see step v in Fig. 2) are compared, hence $\vec{\delta}$ is obtained. $\beta$ is the angle between the normal to the mean plane of the discontinuity and the core axis.

\subsubsection{Quantitative evaluation of the in-plane shift $\vec{\delta}$ by image cor- relation}

Let us consider two sides of an open discontinuity, assumed to be parallel in situ. It is also assumed that correlations between both sides are visible: 
typically, some similar patterns in the topography can be observed on both surfaces. Some patterns may also be identified because of similar colors (like oxidized parts). Estimate of the in-plane shift $\vec{\delta}$ can be done using either maps of the topography, or any top view images resulting from any technique (like photos) where the similar patterns to be correlated are visible. In Fig. 2, the procedure used to deduce $\delta$ from the topography measurement is presented. Once the measurement of the topography is known (step i) (e.g. using a profiler, or other techniques that can produce scaled three dimensional data), a change of coordinates is done, performing a rotation of the initial axes, in order to get the so called $x-y$ plane aligned with the mean plane of the surface (Fig. 2, step ii). Then a rotation of $180^{\circ}$ around the $y$-axis of one of the surfaces is performed (Fig. 2, step iii). Then it is assumed that only a 1D rotation around the $z$ - axis and a 2D translation of the top surface axes (Fig. 2, step iv) are required to position the facing asperities at the same coordinates. This $2 \mathrm{D}$ transformation can theoretically be obtained in several ways: either minimization of volume difference between similar zones, or inter correlation (e.g. Brown (1992); Wilson and Theriot (2006)) of images or surface elevations. However, if both surfaces are not similar enough, with possible outliers in the measurement, we have found that the volume minimization method was not reliable (i.e. clear mismatch of the facing patterns). A simple method to implement is to inspect visually both maps of elevation as images and to select $N$ pairs of points of coordinates $\left(x_{i}^{B}, y_{i}^{B}\right)$ and $\left(x_{i}^{T}, y_{i}^{T}\right)$ (one from each image), that are confidently identified as corresponding points of two homologous asperities. The isometry $\Omega$ to be applied is determined by a least square minimization, i.e. minimization of $\sum_{i=1}^{N}\left[\sqrt{\left(x_{i}^{B}-x_{i}^{T^{\prime}}\right)^{2}+\left(y_{i}^{B}-y_{i}^{T^{\prime}}\right)^{2}}\right]$ where $\left(x_{i}^{T^{\prime}}, y_{i}^{T^{\prime}}\right)=\Omega\left(x_{i}^{T}, y_{i}^{T}\right)$. Then, the in-plane projection of the intercept between the core an the discontinuity are actually fitted with an identical ellipse for both sides (Fig. 2, step v). Once the transformation is made, each top and bottom corresponding point of the elliptic boundaries is shifted of $\vec{\delta}$. By comparing the positions of both fitted ellipses, an estimate of the variability of the field $\vec{\delta}$ can be estimated and, if it is uniform (corresponding therefore to a relative translation of the sides without rotation), the in-plane shift $\delta$ is obtained. 


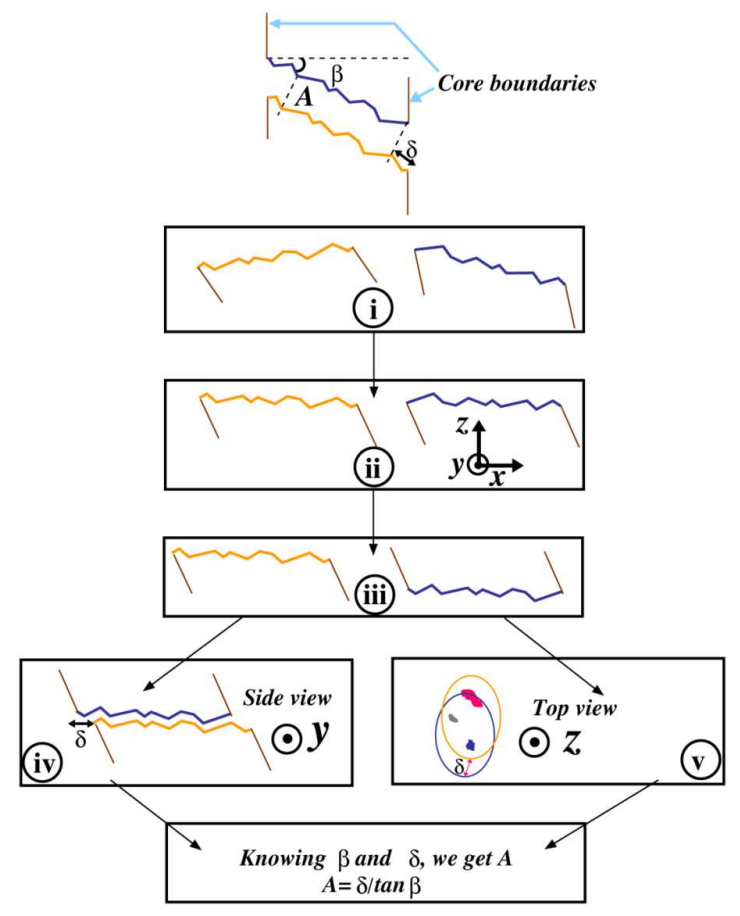

Figure 2: The displacement is assumed here to be normal to the mean plane of the discontinuity. The diagram shows the major five steps of the processing. i: Measurement of the surface topographies. ii: Detrending by rotation of the surfaces; after this rotation, the mean surface plane, computed by a least mean square method, defines the $(x, y)$ plane. iii: Rotation of $180^{\circ}$ of the top surface around the $y$-axis. iv: Asperity correlation and linear transformation (in-plane rotation and translation) to adjust the two sides one against the other; this step requires similarities between both sides. v: Fit of the elliptic boundaries of the sample cores. In this processing, the mean planes of each discontinuity sides were assumed to be initially parallel, like it is the case for F9.

\subsubsection{Application of the method to a real discontinuity from Draix borehole}

The natural discontinuity which is studied here is the one called F9, extracted from a drill in marl at Draix (French Alps) (see paper 1). F9 is an open discontinuity in the sense that no infill material was observed and no contact zone between the top and bottom parts of the discontinuity is known at the core scale. 

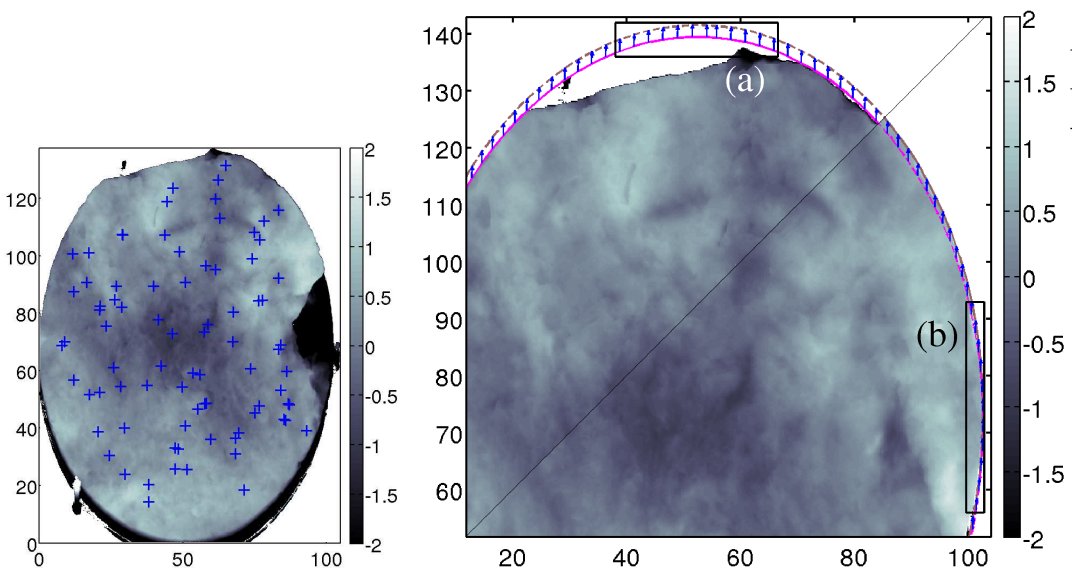

(a)

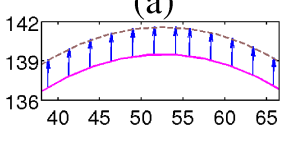

(b)

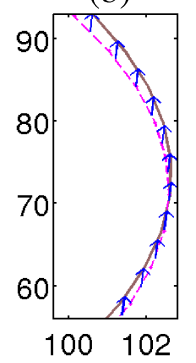

Figure 3: Left: Points selected on the bottom surface of F9. Points corresponding to the similar asperities were also selected on the F9 top surface. Using these points, cross-correlation was done in order to positione both surfaces relatively to one another. Middle: Topography of both sides of the discontinuity F9. Composite picture formed of two parts: topography of the bottom side shown on the top left, topography of top side on the bottom right, positioned by maximization of the cross correlations. It shows that similar patterns are at the same coordinates while the ellipses fitting both elliptical boundaries are shifted by $\vec{\delta}$. Right: Blow up (a) and (b) show that $\vec{\delta}$ is approximatively homogeneous.

The topography of both sides of F9 is known from the measurement with an optical profiler (paper 1). Actually, F9 shows very similar top and bottom surfaces. Once step ii and iii are performed, one searches the best transformation to be applied so that similar pattern are facing. The transformation obtained by selecting pairs of points (Fig. ) provided a good match of the similar patterns, as shown in Fig. 3. The elliptical boundaries of the surfaces can indeed be fitted with an ellipse of identical size for the top and bottom sides, but with different center and orientation. Comparing the position of those ellipses once the corresponding patterns match, it was found that the transformation to be applied to transform one of the ellipse to the other was mostly a translation and a negligible rotation: $\vec{\delta}$ is approximatively uniform. The translation was determined as equal to $\delta=2.085 \pm 0.05 \mathrm{~mm}$.

As no striation was seen on the surfaces, it was deduced that fractures opened in mode I, with a displacement normal to the fracture plane. This mode is coherent with a fracturing due to a pore pressure increase (hydraulic fracturing). Many other mechanisms can also lead to such tensile fractures, as local contraction of the material due e.g. to temperature change, drying, chemical change. The oxidation shows that independently of the cause of the 
fracture, water circulated in it after its creation. The angle $\beta$ was measured as $\beta=42^{\circ}$, which implies $A_{F_{9}}=\delta / \tan \beta \simeq 2.3 \pm 0.1 \mathrm{~mm}$.

The final step is to reconstruct the volumetric aperture, knowing the average aperture, as well as the fluctuation of both surface elevations around their mean plane. Hence both surface elevations are interpolated on the same $(x-y)$ grid (using transformed coordinates, after steps i to iii shown in Fig 2 are performed) and finally the detailed aperture field of F9 shown in Fig. 4 is obtained. In this figure, it can be seen that the aperture in the central zone is about $0.2 \mathrm{~mm}$ larger than on the border of the core, and that some small zones of about $3 \mathrm{~mm}^{3}$ show a locally larger aperture than the one in the surroundings.

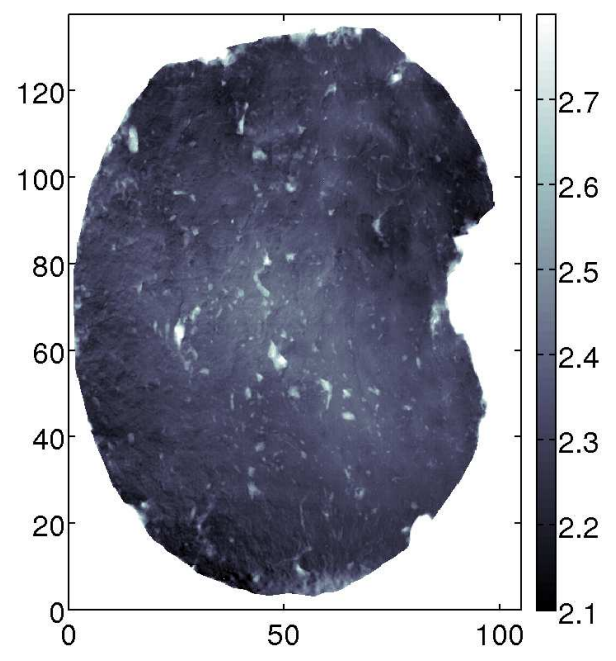

Figure 4: Aperture of discontinuity F9 issued from the laser profiler topography measurement and reconstruction from image correlation. Units are in mm.

\subsection{Reconstruction of the thickness of a sealed discon- tinuity}

For a sealed discontinuity, which has been isolated from the matrix, the aperture can be visually appreciated, but remains to be fully quantified. This can be achieved by measuring the elevation on each side of the sample, so as to deduce its thickness by appropriate transformations (Fig. 5). A critical condition for such a procedure to provide meaningful results is that both topography measurements share a common reference frame, which may be adequately done by wedging the vein into a rigid box of known geometry (Figs. 5, 6 of this paper and Fig. 6 in paper 1). First, the topography 
of one surface (called top, say) is determined together with the boundaries of the box $\left(A_{1} B_{1} C_{1} D_{1}\right)$. Second, the box is flipped upside down and the topography of the other surface (bottom) is similarly measured. It is then possible to transform coordinates from the global frame - given by the profiler - to the local frames $\mathcal{R}_{1}$ and $\mathcal{R}_{2}$, which are associated with, the top and bottom boundaries of the box, as shown in Figs. 5 and 6 . To do so, a precise knowledge of the box geometry is also mandatory. The use of a rectangular parallelepiped box greatly eases this procedure (see practical protocol in Appendix 6). Once the topography is known in frames $\mathcal{R}_{1}$ and $\mathcal{R}_{2}$, as well as the relative position of both these frame, the aperture can finally be obtained.

This procedure was applied to a natural discontinuity, called C14 in paper 1, extracted from a drill at Draix (French Alps). C14 refers to a discontinuity sealed with calcite, in-situ embedded into marl, which was studied after removing the surrounding bulk marl. This discontinuity was then wedged in a box, and the topography of each side was measured with an optical profiler (Fig. 6 in paper 1), allowing to reconstruct its thickness geometry, shown in Figure 7.

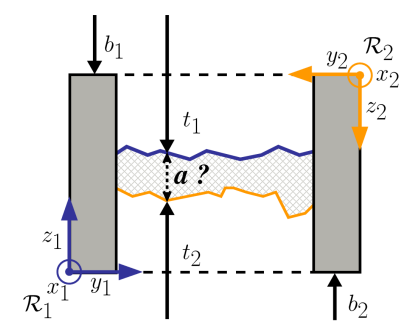

Figure 5: Schematic side view of the sample. By measuring the topography of both surfaces, its thickness can be deduced, see text for details.

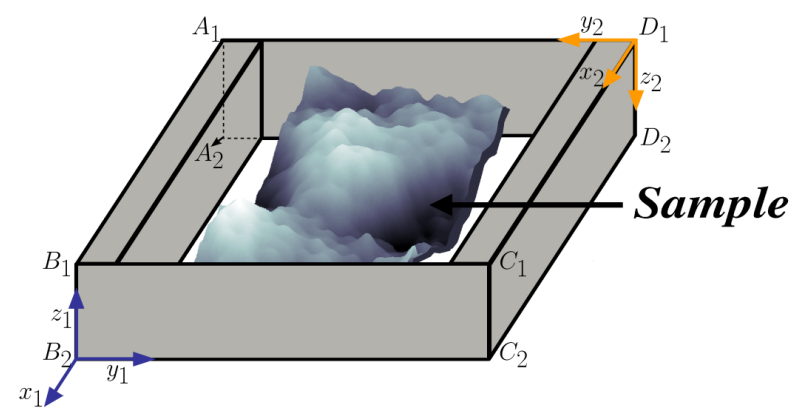

Figure 6: Drawing of a sealed discontinuity wedged in a rigid frame (box). 
The error of the in-plane relative positioning between frames $\mathcal{R}_{1}$ and $\mathcal{R}_{2}$ and translates into an error on the discontinuity aperture, which has been estimated by two independent reconstructions. This error is on average $0.2 \mathrm{~mm}$, and increases with the slope. Most of the uncertainty on the aperture comes from the boundaries of the box not being exactly rigid and orthogonal, which can be improved by using a box designed better than for this exploratory test.

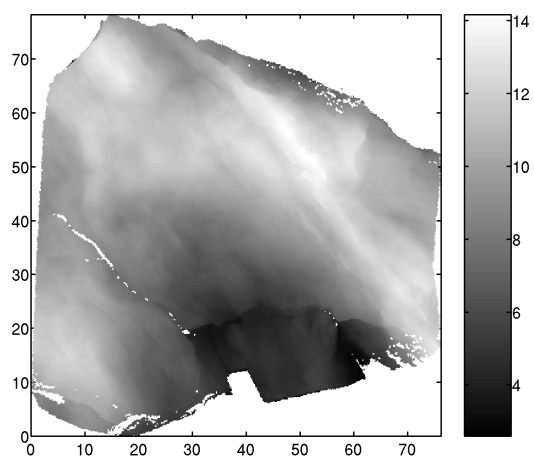

Figure 7: Reconstructed aperture field of C14. Units are in mm.

\section{Statistical characterization of the apertures}

\subsection{Characterization of the aperture morphology of an open discontinuity}

The aperture field of F9 ranges between $2 \mathrm{~mm}$ and $3 \mathrm{~mm}$ (Fig. 4). Its probability density function (Fig. 8, E) is approximately normal (Fig. 8, F) with a standard deviation of $0.08 \mathrm{~mm}$ around the mean aperture $A=2.3 \mathrm{~mm}$. The standard deviation of the aperture is about five times lower than the standard deviation of the elevation of each side, which is $0.4 \mathrm{~mm}$ (paper 1 , recalled in Fig. 8, A, B, C, D). This results already shows that the surface topographies are highly correlated. Further comparison between the top and bottom surface topographies is done by analyzing their scaling properties. 


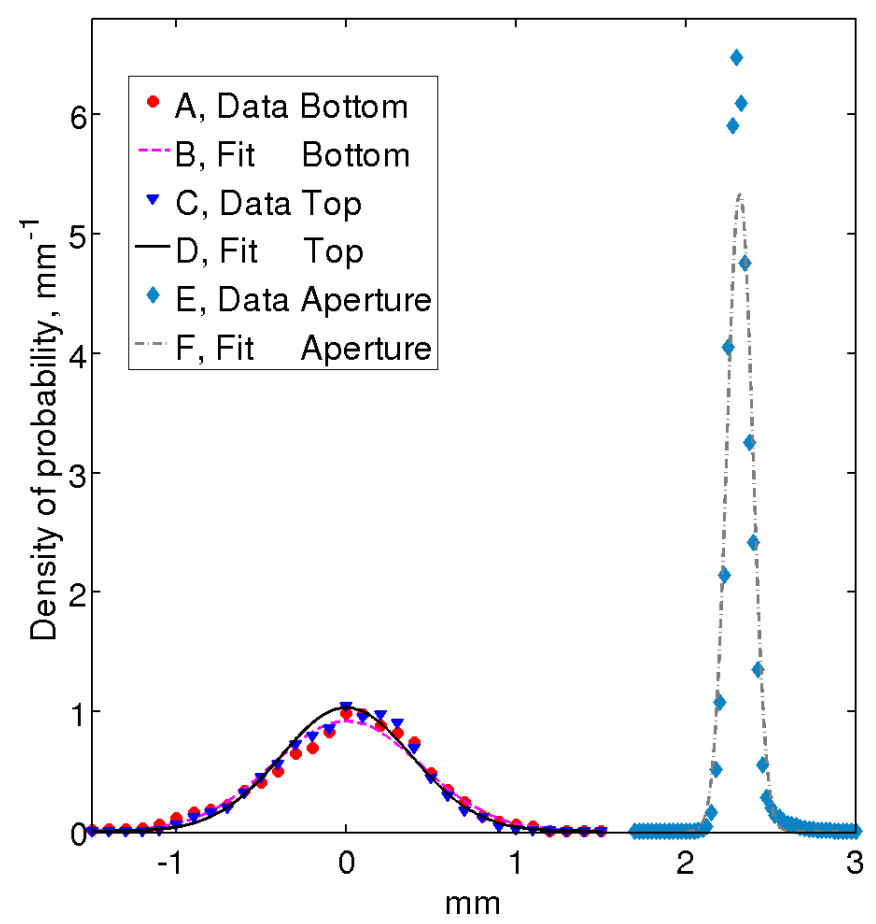

Figure 8: Probability density functions of the elevation of the bottom (A) and top sides $(\mathrm{C})$, and aperture (E) of F9, and their normal probability fits (B, $\mathrm{D}, \mathrm{F}$ ). For A, B, C, D, mean value is $0 \mathrm{~mm}$ and RMS (Root Mean Square) is about $0.4 \mathrm{~mm}$. For E, F, mean value is $2.3 \mathrm{~mm}$ and RMS is about $0.08 \mathrm{~mm}$. The measurements for the plots A, B, C, D are described in paper 1 .

The scaling law of the aperture has been determined exactly with the same autocorrelation expression as for the topographies (see paper 1, section 5),

$$
C_{2}(\vec{\Delta})=\sqrt{\left\langle[f(\overrightarrow{O M}+\vec{\Delta})-f(\overrightarrow{O M})]^{2}\right\rangle}
$$

where $f$ is the function to be analyzed (here, the aperture), $\vec{\Delta}\left(\Delta_{x}, \Delta_{y}\right)$ the lag vector (i.e. characteristic distance at which the correlation is computed), \langle\rangle indicates that the average is computed over all the possible spatial points $M$ so that $z(\overrightarrow{O M})$ and $z(\overrightarrow{O M}+\vec{\Delta})$ are defined, and $\Delta_{0}$ is here chosen to be $1 \mathrm{~mm}$. The self-affinity was checked in the same way as in paper 1 , i.e. by plotting $C_{2}(\Delta)$ as a function of $\Delta$ in a log-log representation: if such a plot is linear, the slope provides a direct determination of the Hurst exponent. This has been plotted in 1D (Fig. 9), for given directions $\theta$, defined as the 
angle between the $x$-axis and $\vec{\Delta}$, and in 2D (Fig. 10), in order to check the isotropy of the scaling law. Figure 9 shows that the scaling law property of the discontinuity aperture, plotted for two directions, is rather different from that of each side, whose scaling curves (A, B, C, D) are recalled for comparison. While those were observed to be self-affine at least from $0.04 \mathrm{~mm}$ to $7 \mathrm{~mm}$ (paper 1), the aperture (E, F) exhibits a self-affine property only on a very short range of scales (from $0.04 \mathrm{~mm}$ to $0.8 \mathrm{~mm}$ ). At larger scales, the autocorrelation $C_{2}$ for the F9 aperture tends to saturate, with a moderate crossover where the transition between both regimes occurs. Similar analytic and numerical scaling curves were discussed e.g. by Brown (1995); Plouraboué et al. (1995); Glover et al. (1998); Ogilvie et al. (2006); Méheust and Schmittbuhl (2003); Auradou et al. (2006), who studied the aperture obtained from two self-affine surfaces matching at large scales and independent at small scales. In our case, the mismatch at small scales is isotropic and would correspond to small scale surface damaging, while the large scale match probably reflects the initial match of the fracture boundaries. Here, the Hurst exponent characterizing the self-affinity at small scales is difficult to robustly estimate, due to the very short range where the self-affinity can itself be observed. The fit proposed in Fig. 9 has a slope (Hurst exponent) of 0.7. This is in accordance with the results presented in paper 1 , where it was shown that both sides of F9 have a self-affine geometry characterized by a Hurst exponent of 0.7 to 0.75 . Figure 10, left, is a 2D plot of $\log _{10}\left[C_{2}(\Delta) / \Delta_{0}\right]$ as a function of the polar coordinates $\left(\log _{10}\left(\Delta / l_{p}\right), \theta\right)\left(l_{p}=0.04 \mathrm{~mm}\right)$. It shows that the scaling properties of the aperture are roughly the same in any direction, which was also the case for its surface. 


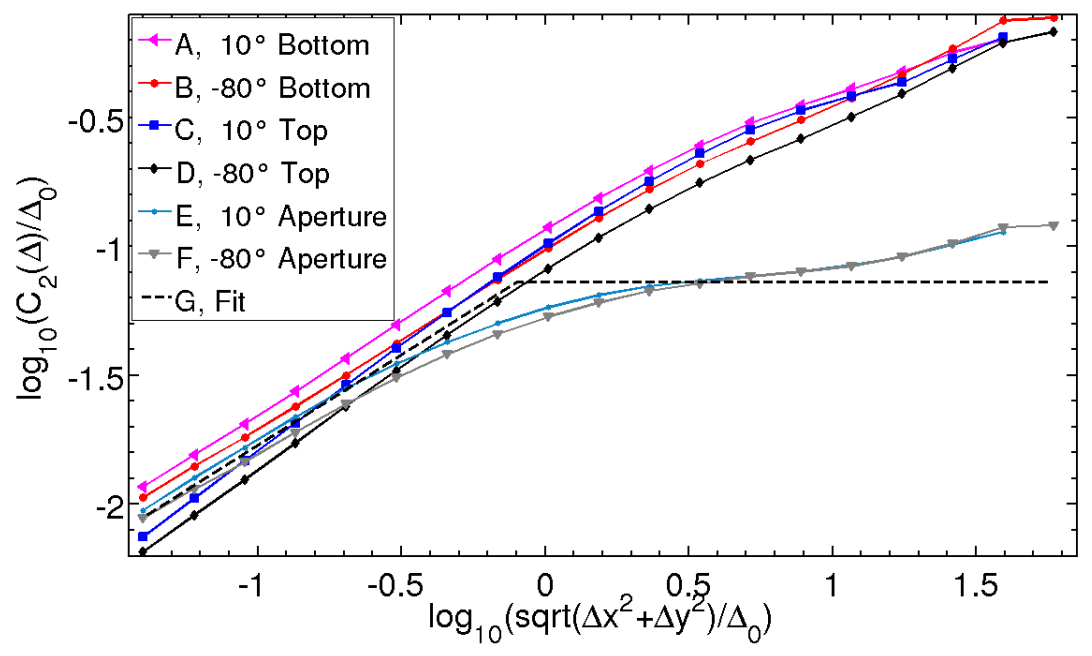

Figure 9: Plot of $\log _{10}\left[C_{2}(\Delta) / \Delta_{0}\right]$ as a function of $\log _{10}\left[\Delta / \Delta_{0}\right]$, for discontinuity $\mathrm{F} 9$, in directions $\theta$ equal to $10^{\circ}$ and $-80^{\circ}$, for the bottom side $(\mathrm{A}, \mathrm{B})$, top side $(C, D)$, and aperture $(\mathrm{E}, \mathrm{F})$, respectively. The measurements for the plots A, B, C, D are described in paper 1. The fit curve shows the suggested model for the F9 aperture: it is self-affine from $\Delta=0.04 \mathrm{~mm}$ to $\Delta=0.8 \mathrm{~mm}$ with a slope of 0.7 and $C_{2}(0.1 \mathrm{~mm})=17 \mu \mathrm{m}$; then for $\Delta$ values higher than $0.8 \mathrm{~mm}$ it reaches a plateau characterized with $C_{2}(\Delta>0.8 \mathrm{~mm})=0.07 \mathrm{~mm}$ 


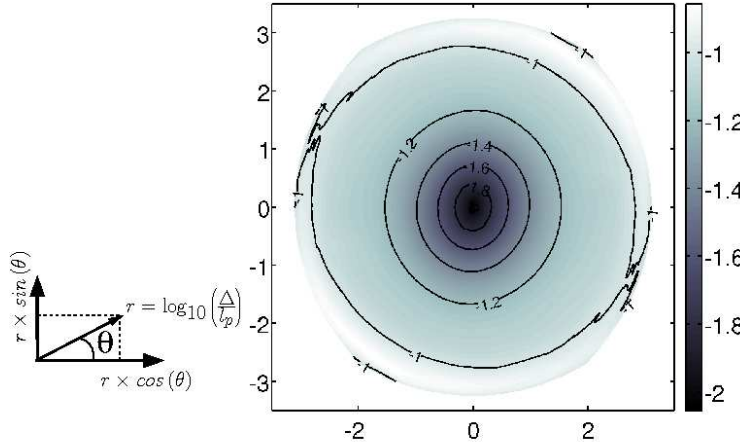

F9

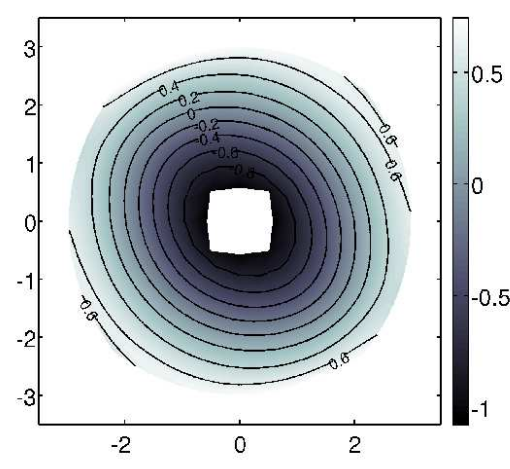

$\mathrm{C} 14$

Figure 10: Representation of $\log _{10}\left[C_{2}(\Delta) / \Delta_{0}\right]$ as a function of the polar coordinates $\left(\log _{10}\left(\Delta / l_{p}\right), \theta\right)$, where $l_{p}$ is $0.04 \mathrm{~mm}$, computed for the apertures F9 (left) and C14 (right). For C9 and C14, the isovalue lines are a family of slightly elongated circles, showing that both apertures are isotropic at first order. Note that, at second order, C14 is slightly more anisotropic; and also that $C_{2}$ values are higher for C14 than for F9. These lines are regularly distributed for $\mathrm{C} 14$, showing that the aperture is self-affine over all the observed ranges, while F9 aperture shows only a self-affine law at very small scales.

\subsection{Scaling law of the thickness of the sealed disconti- nuity}

The calcite joint studied, C14, has a thickness ranging from about $3 \mathrm{~mm}$ to $14 \mathrm{~mm}$. This thickness (shown in Fig. 7), mostly around $1 \mathrm{~cm}$, is relatively variable due to the anastomose of multiple calcite layers (see Fig. 5 in paper 1).

$C_{2}$ was computed for aperture C14, and Fig. 11 shows the 1D plot of $\log _{10}\left[C_{2}(\Delta) / \Delta_{0}\right]$ as a function of $\log _{10}\left[\Delta / \Delta_{0}\right]$ for two directions (hereafter demonstrated as being close to the directions showing the largest anisotropy): $\theta=-35^{\circ}$ and $\theta=55^{\circ}$. A linear trend can be observed from $0.3 \mathrm{~mm}$ to $25 \mathrm{~mm}$, which means that a self-affine law can be fitted. As shown by Fig. 12, $C_{2}$ for C14 is slightly anisotropic, as was the case for its surfaces, with $C_{2}\left(\Delta_{0}\right)$ values progressively varying (amplitude of \pm 0.08 ) according to the direction, around $0.34 \mathrm{~mm}$, with extremal values around $\theta$ equal to $-50^{\circ}$ and $40^{\circ}$. The Hurst exponent also shows an anisotropy (however less pronounced), as it varies around 0.78 according to the direction, by \pm 0.04 . Note that the sides and the aperture measurements are oriented relatively to one another (see section 2.2), so that the directions of anisotropy can be compared between 
both surfaces and the aperture. The difference to an isotropic self-affine surface can be fully observed in Fig 10, right, from the fact that the isovalues are elongated circles. It can also be noticed that the anisotropy direction slightly evolves according to the range of scales. Ignoring these second order effects leads to a global Hurst exponent of about 0.8 for the aperture and 0.75 for the individual sides, whose scaling curves (Fig. 11 A, B, C, D) are recalled for comparison (see paper 1). $C_{2}\left(\Delta_{0}\right)$ of aperture C14 is however shown to be larger than the one of its sides (Fig. 12, right), which simply shows that the spatial variability at $1 \mathrm{~mm}$ of the aperture is larger than the variability of the topography of both sides. This is a behavior opposite to what is observed for discontinuity F9, whose aperture shows less fluctuations than its sides.

C14 is an example of discontinuity with two independent sides which are both self-affine with approximately the same Hurst exponent, which provides a thickness which is self-affine over the same range of scales as that of the sides.

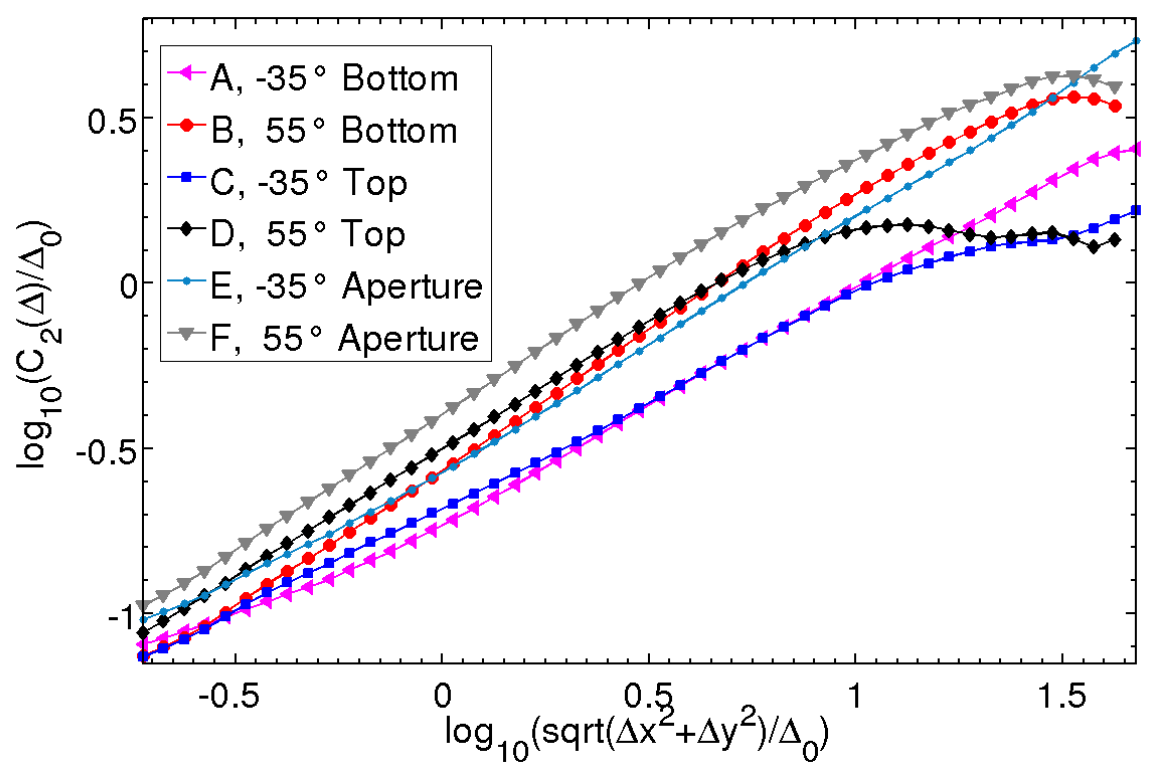

Figure 11: Plot of $\log _{10}\left[C_{2}(\Delta) / \Delta_{0}\right]$ as a function of $\log _{10}\left[\Delta / \Delta_{0}\right]$, for the C14 discontinuity, in directions $\theta$ equal to $-35^{\circ}$ and $55^{\circ}$, for the bottom side (A, $\mathrm{B})$, top side $(\mathrm{C}, \mathrm{D})$, and thickness $(\mathrm{E}, \mathrm{F})$, respectively. The model suggested for the C14 aperture is shown in Fig. 13. The measurements for the plots A, $\mathrm{B}, \mathrm{C}, \mathrm{D}$ are described in paper 1 . 

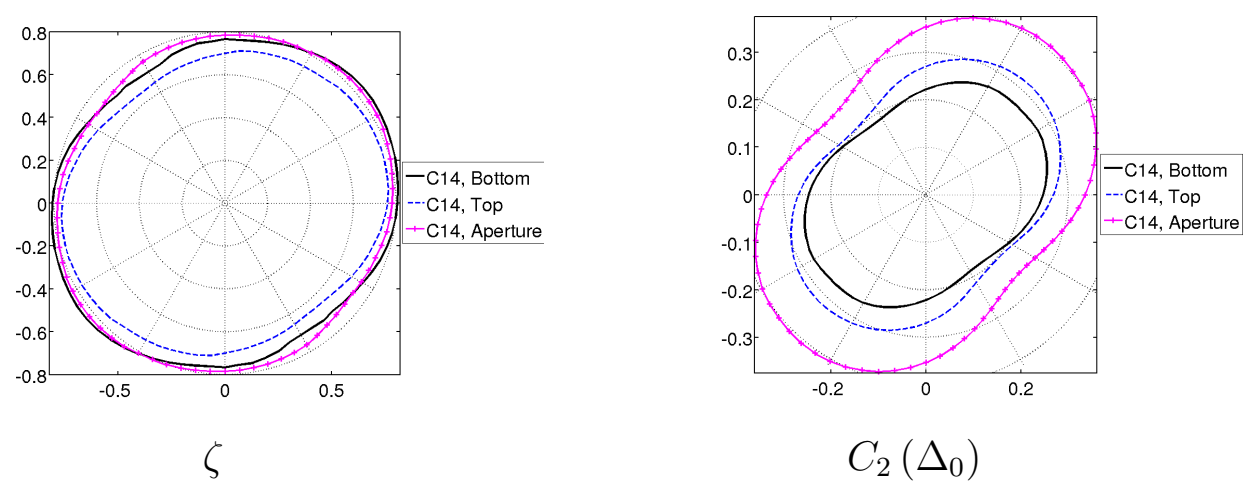

Figure 12: Polar plot of $\zeta$ (left) and $C_{2}\left(\Delta_{0}\right)$ in mm (right) as functions of $\theta$, for the topography of the sides, and aperture, as indicated by the labels, for C14 discontinuity. The ellipsoidal shape of the curves shows how anisotropic the scaling are.

\section{Hydraulic properties of the Draix formation}

Description of the parameters influencing the permeability of a fractured bedrock The aim is to estimate the present permeability $k$ of the Draix fractured bedrock at large scale. Open fractures, like F9, will play a significant role in the permeability. The bedrock being essentially intact marl, the permeability in the perpendicular direction of the mean plane fracture is supposed to be negligible in comparison to the in-plane permeability. An increase of permeability might come from the dissolution of sealed discontinuities of the type previously considered (C14), or even from simple washing of less consolidated clay filled discontinuities (like F6 discontinuity, studied in paper 1), which also exist along the drill. The estimate of the permeability requires the evaluation of the density of open and sealed discontinuities along the core, as well as their orientation. The morphology of the discontinuities also greatly influences the permeability, as was shown, for instance, with numerical simulations of the hydraulic flow, performed on synthetic apertures (e.g. Drazer and Koplik (2002); Al-Yaarubi et al. (2005); Zimmerman and Bodvarsson (1996); Brown (1987); Auradou et al. (2005); Méheust and Schmittbuhl (2001, 2003)). Among the numerically explored aperture models, isotropic rough apertures showing self-affinity at any scale, or at limited scale (e.g. Brown (1987); Zimmerman and Bodvarsson (1996); Méheust and Schmittbuhl (2001, 2003); Neuville et al. (2009a, 2010)), have been compared, in term of permeability, to apertures with flat sides. Those simulations, using the Reynolds equation in the permanent and laminar regimes, under the lubrication approximation (e.g. Zimmerman and 
Bodvarsson (1996)), have shown that, if $\sigma / A$ is small enough (where $\sigma$ is the root mean square of the aperture field), the cubic law (e.g. Zimmerman and Main (2004))

$$
k=\frac{N A^{3}}{12}
$$

is valid, where $N$ is the density of isotropic fractures (number of fractures per length unit), of same orientation and mean aperture $A$. The study of the aperture morphology of the open and sealed discontinuities can therefore be used to determine if that cubic law can be applied to Draix. It is first needed to extrapolate, to the scale of the formation the statistical properties observed at the sample scale. Since the determined aperture correlations are almost isotropic, the associated permeability is isotropic along the discontinuity mean plane. However, since most observed fractures display quite similar directions, the permeability in the direction normal to these fractures would rather be the one of the bulk marl.

Hydraulic contribution of the open discontinuities F9 is assumed to have an aperture representative of all the open fractures in the formation. It was shown that the average aperture is about $A_{F_{9}}=2.3 \mathrm{~mm}$, with very little variations around that mean (Figs. 4 and 8): $\sigma_{F_{9}} / A_{F_{9}}<0.04$. The aperture was also shown to follow a self-affine law at very small scales (less than $1 \mathrm{~cm}$ ), and to be statistically invariant at larger scales. It also means that most contribution on the roughness comes from the short scales of the morphology. Méheust and Schmittbuhl (2003) showed that the hydraulic behavior, under lubrication approximation, for fracture apertures with such scaling characteristics are controlled by the small scales. Therefore, the behavior of F9 can be deduced from numerical simulations performed over numerous self-affine apertures (Neuville et al., 2010), showing $\sigma / A<0.04$ : it was shown that the hydraulic behavior computed for a fracture with such a small $\sigma / A$ ratio can be approximated by that of a parallel plate model with aperture $A$, with a precision better than $99 \%$ on the hydraulic aperture.

Counting the number of open discontinuities visible along the core for fractured unit 2, which is the unit where open discontinuities are numerous, we get approximately four discontinuities over four meters, i.e. $N_{o} \simeq 1$ open discontinuity per meter. From equation (2), the permeability is thus of order $k=10^{-9} \mathrm{~m}^{2}$ in the direction of the fractured bedrock. That means that unit 2 is highly permeable. Apertures of some of the open fracture might however be different from $2.3 \mathrm{~mm}$, with a slightly different orientation, which might result into a different permeability.

Potential hydraulic contribution of sealed discontinuities and of discontinuities containing debris and clay. Using calcite vein C14, 
the hydraulic permeability increase if such calcite veins were dissolved can be estimated. In subsection 3.2 it was observed that $\mathrm{C} 14$ calcite thickness (i.e. the potential discontinuity aperture) follows a self-affine law with Hurst exponent of about 0.8 , at least up to scales of order $25 \mathrm{~mm}$, with a prefactor given by $C_{2}\left(\Delta_{0}\right) \simeq 0.3 \mathrm{~mm}$. As the C14 sample size is only about $6 \times 7 \mathrm{~cm}^{2}$, the $C_{2}$ could not be computed over about half size of the sample, i.e. about $3 \mathrm{~cm}$. As shown by studies about the morphology of natural fractures, it is known, that at large scales, the aperture tends to be statistically invariant (e.g. Plouraboué et al. (1995); Isakov et al. (2001)), showing a constant autocorrelation value $C_{2}\left(\Delta_{l}\right) . C_{2}\left(\Delta_{l}\right)$ should be of the order of the root mean square of the aperture, which is statistically smaller than the average aperture (otherwise, the aperture field would exhibit negative values). Assuming that the mean aperture observed at the sample scale (of order $1 \mathrm{~cm}$ ) is the same at larger scale, it means that the scaling law is at most valid until $C_{2}\left(\Delta_{l}\right)=$ $1 \mathrm{~cm}$. Thus the relationship $\log _{10}\left[C_{2}\left(\Delta_{l}\right) / \Delta_{0}\right]=-0.5+0.8 \log _{10}\left[\Delta_{l} / \Delta_{0}\right]$ provides $\Delta_{l}=75 \mathrm{~mm}$ (see abscissa of point $\mathrm{S}$ in Fig. 13). At scales larger than this correlation length, $\Delta_{l}=75 \mathrm{~mm}, C_{2}(\Delta)$ should be essentially constant.

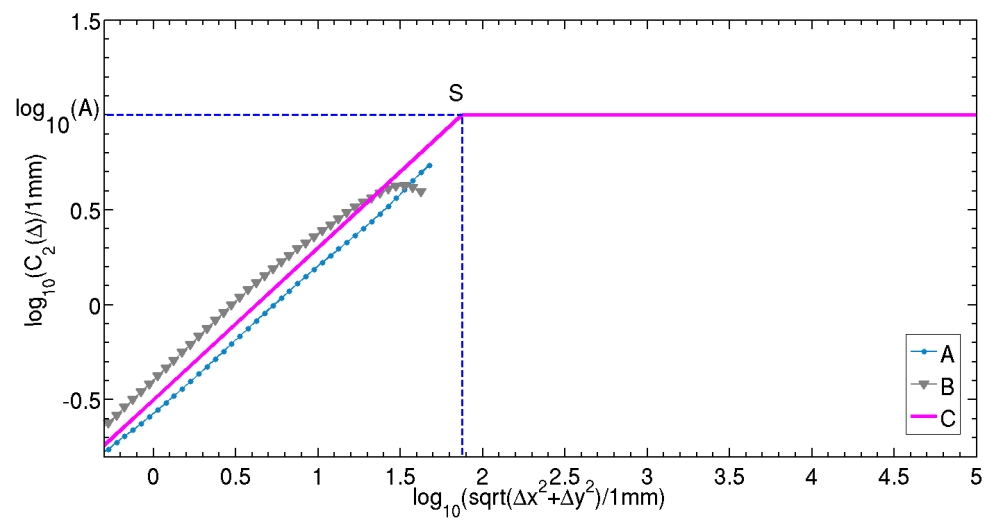

Figure 13: Plot of $\log _{10}\left[C_{2}(\Delta) / \Delta_{0}\right]$ as a function of $\log _{10}\left[\Delta / \Delta_{0}\right]$ for $\theta$ equal to $-35^{\circ}(\mathrm{A}), 55^{\circ}$ (B) for the aperture C14. Plot $\mathrm{C}$ shows the deduced geometrical model applied at small and large scales. Point $\mathrm{S}$ is the intersection between the scaling law $\log _{10}\left[C_{2}(\Delta) / \Delta_{0}\right]=-0.5+0.8 \log _{10}\left[\Delta / \Delta_{0}\right]$ and $C_{2}(\Delta)=A=1 \mathrm{~cm}$. Assuming that the fluctuation of the aperture cannot be larger than the average $A$, the self-affine law is considered to show a cutoff at scales larger than the correlation length $\Delta_{l}=75 \mathrm{~mm}$, which is the abscissa of S. [SI PB DE PLACE, LE MODELE PEUT ETRE PLACE SUR LA FIGURE 11]

The hydraulic behavior is determined for a self-affine variable fracture (i.e., for scales smaller than $75 \mathrm{~mm}$ ), using the Reynolds equation in the per- 
manent and laminar regime, with the lubrication approximation (e.g. Zimmerman and Bodvarsson (1996)), solved with finite differences (e.g. Méheust and Schmittbuhl (2000, 2003); Schmittbuhl et al. (2008); Neuville et al. (2009a, 2010)). As no correlations in the fracture aperture are expected above a scale of $75 \mathrm{~mm}$, the hydraulic behavior of the fracture at the formation scale is likely to be identical to that of a self-affine fracture of size $75 \times 75 \mathrm{~mm}^{2}$ (length over width). Figure 14 shows an example of (i) a synthetic aperture field of size $75 \times 75 \mathrm{~mm}^{2}$ with $\zeta=0.8$ and mean aperture $A=1 \mathrm{~cm}$ and (ii) an extract of the aperture C14, of size $52 \mathrm{~mm} \times 58 \mathrm{~mm}$ with $\zeta=0.8$ and $\sigma / A=0.3$. For the synthetic case, the root-mean square aperture variation, $\sigma$, is fixed by the maximum possible value permitting to avoid contact between the fracture sides (which results in this case in $\sigma \simeq 0.46 A)$.

Figure 15 shows the corresponding hydraulic flow, $q$ which is defined defined as the norm of the local hydraulic flux. It is shown in dimensionless units, being normalized by $12 \eta l_{x} / \Delta P$, where $l_{x}=75 \mathrm{~mm}$ is the fracture length, $\eta$ is the dynamic viscosity, and $\Delta P$ is the pressure difference imposed between the inlet and the outlet of the fracture. Due to the spatial variability of the aperture, the hydraulic flow is also variable in space. For those examples, the average hydraulic aperture, defined by $H=<-q \cdot 12 \eta \frac{l_{x}}{\Delta P}>^{1 / 3}$, is for case (i) $H=0.7 \mathrm{~cm}$, and for case (ii) $H=0.9 \mathrm{~cm}$, which means that those fractures are less permeable than parallel plates separated by a constant aperture $A=1 \mathrm{~cm}$. From studies previously made on numerous self-affine fractures (e.g. Brown (1987); Méheust and Schmittbuhl (2001); Neuville et al. (2010)), it is known that the hydraulic aperture for a fracture with $\sigma / A>0.45$ and $A=1 \mathrm{~cm}$ is likely to be $H \simeq 0.9 \mathrm{~cm}$ under lubrication hypothesis. Counting the number of sealed discontinuities visible on the core for fractured unit 1, which is the unit where sealed discontinuities are numerous, we get approximately five discontinuities over five meters, i.e. a density $N_{s}^{\max } \simeq 1$ sealed discontinuity per meter. However it is not sure that all the fractures will be re-open at the same time. If only one such fracture re-open along the core in unit 1 , then $N_{s}^{1}=0.2$ sealed discontinuity per meter, which implies $k=10^{-8} \mathrm{~m}^{2}$ in the direction of the fractured marl. This value is significantly larger than the permeability of he open fracture $\left(k=10^{-9}\right)$. As sealed discontinuities show a multiple layer structure (see section 3 in paper 1), one could also conceive that only part of the layers would be dissolves, providing thinner aperture. 

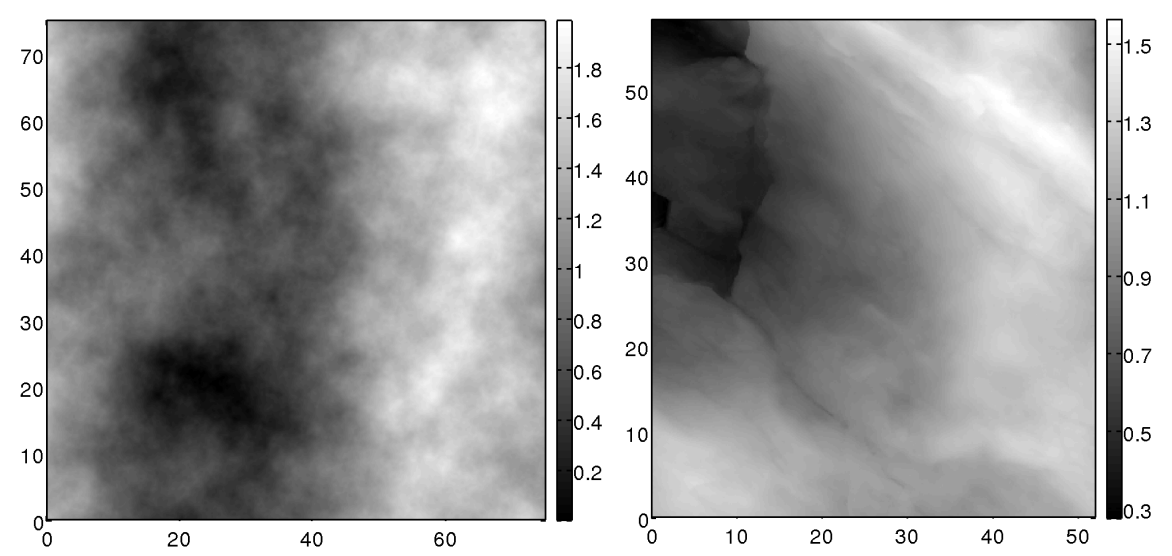

Figure 14: Square map of (i) a synthetic aperture field with $\zeta=0.8, \sigma=$ $0.46 \mathrm{~cm}$, and $A=1 \mathrm{~cm}$ and (ii) an extract of the aperture field C14, with $\zeta=0.8, \sigma / A=0.3$. The plane coordinates are in $\mathrm{mm}$ and the aperture field is shown in $\mathrm{cm}$. Dark areas correspond to thinner apertures while light areas correspond to larger apertures.
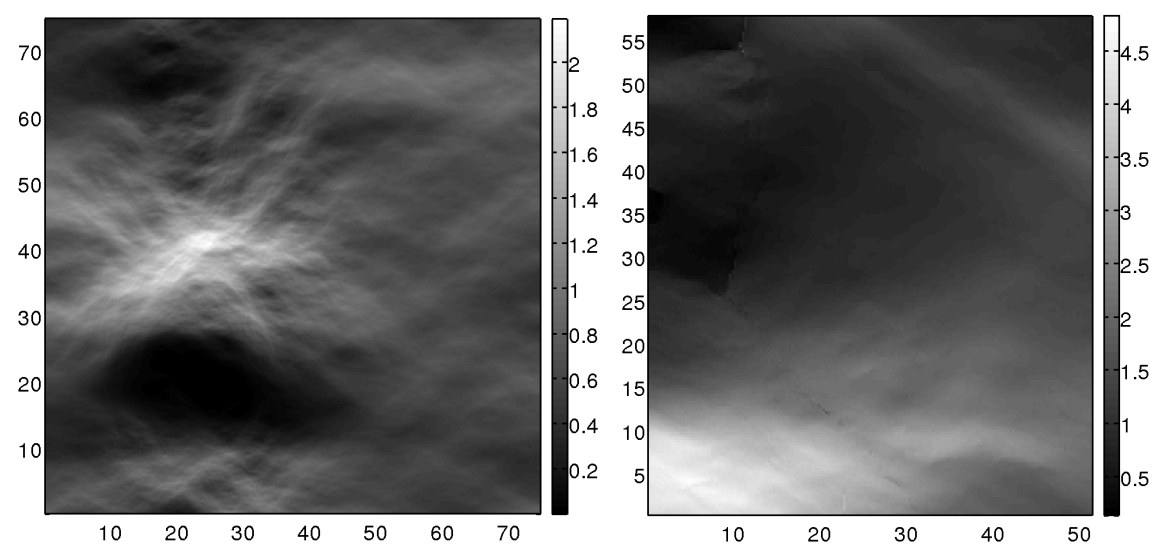

Figure 15: Map of the normalized hydraulic flow computed, from (i) the synthetic aperture field shown in Fig. 14, and (ii) the real field shown in Fig. 14. The plane coordinates are in $\mathrm{mm}$ and the hydraulic flow field in arbitrary units (depending on the pressure gradient). The average hydraulic aperture of these fields are (i) $H=0.68 \mathrm{~cm}$ and (ii) $H=0.9 \mathrm{~cm}$. Dark areas correspond to quasi-static fluid while light areas correspond to larger hydraulic flow. 


\section{Conclusion}

Two techniques for the aperture reconstruction of discontinuities have been developed. The first one, adapted to fully open discontinuities at the scale of the core log, is based on correlating the detailed morphology of their sides. Using this method, the aperture of one of the open discontinuities located in the marl bedrock of Draix has been determined, together with invariance properties. The second one is adapted to sealed discontinuities. The thickness of a such one discontinuity was also computed using measurements of the surface elevations with a laser profiler. The relative spatial positioning of the top and bottom elevation measurements was necessary for reconstructing the thickness of the vein, and was ensured by docking the rock sample into a rigid frame with a known geometry. The ratio between the amplitude of the roughness and the mean aperture was found to be very small for the open discontinuity, contrary to what was obtained fore the sealed discontinuity. The scaling laws of the aperture and thickness of respectively the open and sealed discontinuities were also obtained. The thickness of the vein is selfaffine, with similar Hurst and prefactor values as the ones of its sides. On the contrary, the open discontinuity presents two non independent self-affine surfaces, leading to an aperture which is only self-affine at very small scales and uncorrelated at larger scales. From these geometrical properties, macroscopic hydraulic transport properties of open and sealed discontinuities could be assessed using, in the first case, a simple planar fracture model, and, in the second case, finite element simulations for self-affine apertures. This allowed us to come to an estimate of macroscopic permeability values for these sets of discontinuities: $k=10^{-9} \mathrm{~m}^{2}$ for the opened ones, and potentially $k=10^{-8} \mathrm{~m}^{2}$ for the ones currently sealed, in case the material inside would dissolved or washed away. It means that the fractured marl is potentially highly permeable.

The authors would like to thank José Ortega for his help for the geological description of the core. We also thank Jean-Phillipe Malet and Alexandre Remaître for useful indications about Draix field observations, as well as Grégory Bièvre for helpful information about the SC1 drilling.

\section{Appendix: Experimental protocol to change frames when measuring the aperture using the topography of a sealed discontinuity}

This is an appendix to section 2.2, where it is explained how to reconstruct the aperture of a sealed discontinuity from topography measurement of its surfaces. This is achieved if both topography measurements share a common 
reference frame, which may be adequately done by wedging the vein into a rigid box of known geometry. If the box is a truly rectangular parallelepiped box greatly eases the procedure, that is we will detailed here. First, the topography of one surface (called top, say) is determined together with the boundaries of the box $\left(A_{1} B_{1} C_{1} D_{1}\right.$, Fig. 16 top left). Second, the box is flipped upside down and the topography of the other surface (bottom) is similarly measured (Fig. 16, step i). It is then possible to transform coordinates from the global frame - given by the profiler - to the local frames $\mathcal{R}_{1}$ and $\mathcal{R}_{2}$, where $\mathcal{R}_{1}$ and $\mathcal{R}_{2}$ are the frames associated with, respectively, the top and bottom boundaries of the box, as shown in Figs. 5 and 6 . With a parallelepiped box, it simply remains to bring $A_{1} B_{1} C_{1} D_{1}$ in correspondence with $A_{2} B_{2} C_{2} D_{2}$ (corresponding points of the top surface), thus obtaining new set $\left(x_{p}, y{ }_{p}, z{ }_{p}\right)$ of coordinates for the top surface. This is done with a rotation of $180^{\circ}$ around the $y$ - axis of the top surface (Fig. 16, step ii), followed by an in-plane translation and rotation (Fig. 16, step iii). Finally, knowing the bow thickness, the aperture can be deduced by an appropriate subtraction of the top and bottom measurements.

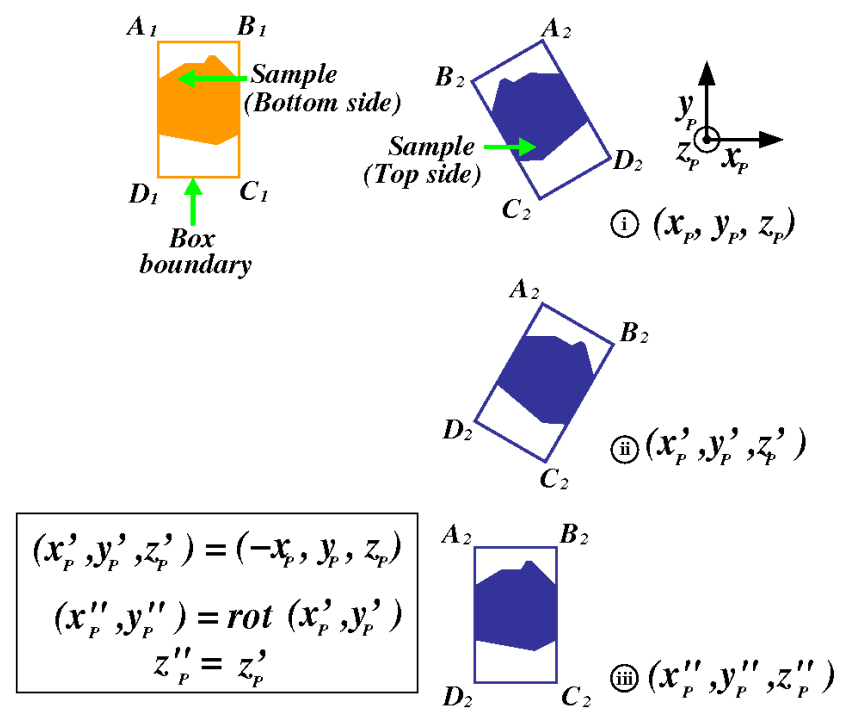

Figure 16: Schematic view of the transformations to be performed, step by step, to bring both measured topographies in a common frame. The sample and the box are viewed from the top (same as the profiler view).

\section{References}

Al-Yaarubi, A., Pain, C., Grattoni, C., and Zimmerman, R. (2005). NavierStokes simulations of fluid flow through a rock fracture. In Faybishenko, B., 
Witherspoon, P., and Gale, J., editors, Dynamics of Fluids and Transport in Fractured Rocks, volume 162, pages 55-64. American Geophysical Union, Washington, DC. AGU Monograph.

Auradou, H., Drazer, G., Boschan, A., Hulin, J.-P., and Koplik, J. (2006). Flow channeling in a single fracture induced by shear displacement. Geothermics, 35(5-6):576-588.

Auradou, H., Drazer, G., Hulin, J. P., and Koplik, J. (2005). Permeability anisotropy induced by the shear displacement of rough fracture walls. Water Resources Research, 41(9):1-10.

Brown, L. G. (1992). Survey of image registration techniques. ACM Computing Surveys, 24(4):325-376.

Brown, S. (1995). Simple mathematical model of a rough fracture. Journal of Geophysical Research, 100(B4):5941-5952.

Brown, S. R. (1987). Fluid flow through rock joints: The effect of surface roughness. Journal of Geophysical Research, 92(B2):1337-1347.

Drazer, G. and Koplik, J. (2002). Transport in rough self-affine fractures. Physical Review E, 66:026303.

Fressard, M., Maquaire, O., Malet, J. P., Klotz, S., and Grandjean, G. (2009). Morpho-structure and triggering conditions of the Laval landslide developed in clay-shales, Draix catchment (South French Alps). In J. P. Malet, A. R. and Bogaard, T., editors, Landslide processes. From geomorphologic mapping to dynamic modelling., pages 111-114, France. CERG, Strasbourg.

Genter, A. and Traineau, H. (1996). Analysis of macroscopic fractures in granite in the HDR geothermal well EPS-1, Soultz-sous-Forêts, france. Journal of Volcanology and Geothermal Research, 72(1-2):121-124.

Gentier, S. S. and Hopkins, D. L. (1997). Mapping fracture aperture as function of normal stress using a combination of casting, image analysis and modeling techniques. International journal of rock mechanics and mining sciences \& geomechanics abstracts, 34(3-4):359.

Glover, P., Matsuki, K., Hikima, R., and Hayashi, K. (1998). Synthetic rough fractures in rocks. Journal of Geophysical Research B: Solid Earth, 103(5):9609-9620.

Hakami, E. and Larsson, E. (1996). Aperture Measurements and Flow Experiments on a Single Natural Fracture.

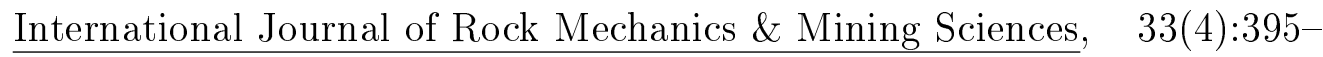
404 .

Isakov, E., Ogilvie, S. R., Taylor, C. W., and Glover, P. W. J. (2001). Fluid 
flow through rough fractures in rocks i: High resolution aperture determinations. Earth and Planetary Science Letters, 191(3-4):267-282.

Lee, H. and Cho, T. (2002). Hydraulic characteristics of rough fractures in linear flow under normal and shear load. Rock Mechanics and Rock Engineering, 35(4):299-318.

Lofi, J., Pezard, P., Loggia, D., Garel, E., Gautier, S., Merry, C., and Bondabou, K. (2009). Petrophysical, borehole geophysics and geological characterization of tectonized clay shales drilled at Draix (southern French Alps). Hydrological Processes, Special issue, hydrology of clay shales and clayey sediments.

Méheust, Y. and Schmittbuhl, J. (2000). Flow enhancement of a rough fracture. GRL, 27:2989-2992.

Méheust, Y. and Schmittbuhl, J. (2001). Geometrical heterogeneities and permeability anisotropy of rough fractures. Journal of Geophysical Research, 106(B2):2089-2012.

Méheust, Y. and Schmittbuhl, J. (2003). Scale effects related to flow in rough fractures. Pure and Applied Geophysics, 160(5-6):1023-1050.

Muralidhar, K. (1990). Flow and transport in single rock fractures. Journal of Fluid Mechanics, 215:481-502. Cambridge University Press.

Neuville, A., Toussaint, R., and Schmittbuhl, J. (2009a). Fracture roughness and thermal exchange: A case study at Soultz-sous-Forêts. Comptes Rendus - Geoscience. In Press.

Neuville, A., Toussaint, R., and Schmittbuhl, J. (2010). Hydro-thermal flows in a self-affine rough fracture. Submitted to Phys. Rev. E.

Neuville, A., Toussaint, R., Schmittbuhl, J., Koehn, D., and Schwarz, J. O. (2009b). Characterization of major discontinuities from borehole cores of the black consolidated marl formation of Draix (French Alps). Hydrological Processes, Special issue, hydrology of clay shales and clayey sediments. Submitted, in revision.

Noiriel, C. (2005). Contribution à la détermination expérimentale et à la modélisation des différents processus contrôlant l'évolution géochimique, structurale et hydrodynamique des roches fissurées carbonatées. PhD thesis, École des Mines de Paris.

Ogilvie, S., Isakov, E., and Glover, P. (2006). Fluid flow through rough fractures in rocks ii: A new matching model for rough rock fractures. Earth and Planetary Science Letters, 241(3-4):454-465.

Plouraboué, F., Hulin, J. P., Roux, S., and Koplik, J. (1998). Numerical study of geometrical dispersion in self-affine rough fracture. Physical Review E, $58(3): 3334-3346$. 
Plouraboué, F., Kurowski, P., Hulin, J.-P., Roux, S., and Schmittbuhl, J. (1995). Aperture of rough cracks. Physical Review E, 51(3):1675-1685.

Pyrak-Nolte, L., Cook, N., and Note, D. (1988). Fluid percolation through single fractures. Geophysical Research Letters, 15(11):1247-1250.

Roux, S., Plouraboué, F., and Hulin, J.-P. (1998). Tracer dispersion in rough open cracks. Transport in Porous Media, 32(1):97-116.

Sakaguchi, K., Tomono, J., Okumura, K., Ogawa, Y., and Matsuki, K. (2008). Asperity height and aperture of an artificial. tensile fracture of metric size. Rock Mechanics and Rock Engineering, 41(2):325-341.

Schmittbuhl, J., Steyer, A., Jouniaux, L., and Toussaint, R. (2008). Fracture morphology and viscous transport. International Journal of Rock Mechanics \& Mining Sciences, $\quad$ 45:422430.

Sharifzadeh, M., Mitani, Y., and Esaki, T. (2008). Rock joint surfaces measurement and analysis of aperture distribution under different normal and shear loading using gis. Rock Mechanics and Rock Engineering, 41(2):299323.

Tsang, Y. and Tsang, C. (1987). Channel model of flow through fractured media. Water Resources Research, 23(3):467-479.

Vicente Silvestre, M. V., Ribeiro e Sousa, L., and Hack, R. (2002). Laboratory study of geomechanical and hydro-mechanical characterisation of discontinuities. News Journal International Society for Rock Mechanics (ISRM), 7(2):915 .

Walkup, G. J. and Horne, R. (1985). Forecasting thermal breakthrough of reinjected water using a dispersion-retention model for tracer test interpretation. In International Symposium - Geothermal Energy, part II, volume 9, pages 369-374. Geothermal Resources Council Transactions. Conference Paper.

Wilson, C. and Theriot, J. (2006). A correlation-based approach to calculate rotation and translation of moving cells. IEEE Transactions on Image Processing, 15(7):1939-1951.

Zimmerman, R. W. and Bodvarsson, G. S. (1996). Hydraulic conductivity of rock fractures. Transport in Porous Media, 23(1):1-30.

Zimmerman, R. W. and Main, I. G. (2004). Mechanics of fluid-saturated rocks, volume 89 of International geophysics series, chapter Hydromechanical behaviour of fractured rocks, pages 363-421. Elsevier, Academic press, London, U.K. 